\title{
LA-UR-21-27062
}

Approved for public release; distribution is unlimited.

Title: $\quad$ Producing the Fission-Product-Isotope File KIDMAN

Author(s): $\quad$ Conlin, Jeremy Lloyd

Seamon, R. E.

Intended for: Report

Issued: 
Disclaimer:

Los Alamos National Laboratory, an affirmative action/equal opportunity employer, is operated by Triad National Security, LLC for the National Nuclear Security Administration of U.S. Department of Energy under contract 89233218CNA000001. By approving this article, the publisher recognizes that the U.S. Government retains nonexclusive, royalty-free license to publish or reproduce the published form of this contribution, or to allow others to do so, for U.S. Government purposes. Los Alamos National Laboratory requests that the publisher identify this article as work performed under the auspices of the U.S. Department of Energy. Los Alamos National Laboratory strongly supports academic freedom and a researcher's right to publish; as an institution, however, the Laboratory does not endorse the viewpoint of a publication or guarantee its technical correctness. 
FROM:

SYMBOL:

SUBJECT:
R. C. Little, X-6, MS
R. E. Seamon, X-6

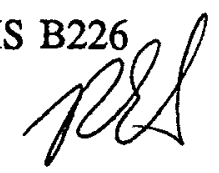

DATE:

15-September-1989

MAIL STOP/TELEPHONE:

B226 / 7-4809

\section{Producing the Fission-Product-Isotope File KIDMAN}

In Ref. 1 I described the CFS file /076997/MYREDSTUFF/KIDMAN, an ACE file for the Monte Carlo code MCNP (Ref. 2) containing transport cross sections for twenty six fission product isotopes from ENDF/B-V. There is a second file associated with KIDMAN, the directory file stored under /076997/MYREDSTUFF/KIDMAND.

I found that Bob MacFarlane, T-2, had processed the evaluations through the RESR and UNRESR modules of NJOY (Ref. 3) to produce PENDF files; there were no ACER files available for any of the isotopes, however. Starting from the PENDF file and the BCD ENDF/B-V tape indicated in Table II of Ref. 1, I used the HEATR and ACER modules of NJOY to produce what are alleged to be Type 3 ACE files which should be useable in MCNP and related checking codes. Nothing would work; the problem was traced to extra record marks which had to be removed using the code FIXACEFILE. I took the opportunity to remove as well the completely unnecessary photon production matrix $(30 \times 20$ zero values) at the end of each file. There is no photon production data for any of the 26 isotopes; I am unable to run the ACER module in such a way that the zeroes would disappear.

The corrected ACE files were processed through ADDXS (Ref. 4) to add charged-particleproduction cross sections for eleven of the isotopes where it was appropriate, and the individual files were merged using your version of Pat Soran's original code CULIT (Ref. 5). Checks were made using XDATAP (Ref. 6), MARK and MRKACR (Ref. 7), and FISHPC (Ref. 4).

The purpose of this memo is to document explicitly how this work was done, or - more truthfully - how I would do the job again knowing what I know now. First, the codes will be listed, then the data decks, and - finally - what problems were encountered.

The codes - -

NJOY: Based on experiences in processing the excited-state data for Dave Madland, I chose to use the binary executeable version of NJOY stored on CFS under /NJOY/89/XNJOY0.

FIXACEFILE: The source for this code is stored on CFS under /076997/MYREDSTUFF /FIXACEFILE. A listing thereof is given in Appendix A. Let CODE=FIXACEFILE; one gets the binary executeable file XCODE using RCFT I=CODE,GO. 
ADDXS: The source is stored on CFS under 1090895/CTSS/ADDXS. One gets the binary executeable XADDXS using RCFT I=ADDXS,GO.

CUL: The source is stored on CFS under /MYREDSTUFF/CUL. One gets the binary executeable $\mathrm{XCUL}$ using RCFT I=CUL,GO.

XDATAP: I use the binary version which has grown out of my collaboration with Harl'O Fisher on the Handbook; it is stored on CFS under /076997/MYREDSTUFF/XDATAP6.

FISHPC: The source is stored on CFS under /090895/CTSS/FISHPC. One gets the binary executeable XFISHPC using RCFT I=FISHPC,LIB=(CFTLIB,SCCFT,CGSCFT),GO.

MARK: The source is stored on CFS under /076997/PGMS/MARK. One gets the binary executeable XMARK using RCFT I=MARK,GO.

MRKACR: The source is stored on CFS under /076997/PGMS/MRKACR. One gets the binary executeable XMRKACR using RCFT I=MRKACR,GO.

The input decks - - -

NJOY: Here is a listing of the file INPUT associated with the NJOY run for Xe-131.

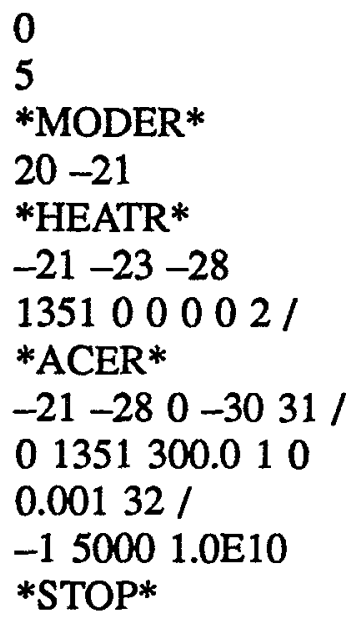

ENDF/B-V BCD input Tape20 was /ENDF/5/A/T/509.

Binary PENDF Tape23 was /PENDF/5/XE/131.

MAT $=1351$

Binary Tape 30 is the pseudo ACE-formatted file - "pseudo" because of those miserable extra record marks.

FIXACEFILE: No input file is needed. Binary Tape30 is the pseudo ACE file to be corrected and binary Tape 31 - a real ACE file - is produced. 
ADDXS: Here is a listing of the TAPE8 input file appropriate for $\operatorname{Pr}^{141}, \mathrm{Nd}^{143}, \mathrm{Nd}^{145}, \mathrm{Nd}^{148}$, $\mathrm{Pm}^{147}, \mathrm{Sm}^{147}, \mathrm{Sm}^{151}, \mathrm{Sm}^{152}$, and $\mathrm{Eu}^{155}$. The format is $2 \mathrm{I} 5$.

$\begin{array}{rr}5 & \\ 203 & 2 \\ 28 & 103 \\ 204 & 1 \\ 104 & \\ 205 & 1 \\ 105 & \\ 206 & 1 \\ 106 & \\ 207 & 2 \\ 22 & 107\end{array}$

CUL: Here is a listing of the CULINP file for a run in which I merged HERFILE containing 25 ZAIDs with file 9832B containing one ZAID $(=63155.50)$. In this run the output file MYFILE became the final file KIDMAN. These lines start in column 1.

\section{MYFILE}

2

HERFILE

9832B

40093.50

42095.50

43099.50

44101.50

44103.50

45105.50

46105.50

46108.50

53135.50

59141.50

61148.50

61149.50

62147.50

62150.50

62151.50

62152.50

63155.50

0. 
FISHPC: Requires that KIDMAN be SWTTCHed to EXTRA and that input file XSIN be one blank line.

MARK and MRKACR: The file INPUT is stored on CFS under /076997/R028/MARKD. For MARK, Tape7 must be the BCD File3 data; for MRKACR Tape7 is an ACE file like KIDMAN.

Additional Remarks - - -

Before I merged the individual ACE files using CUL, I changed the ZAIDs manually using EDIT so that they would have suffix $n n=50$ in ZAID $=$ ZZAAA.nn. Locations $1_{8}$ and $312_{8}$ in file OUTACE from ADDXS were changed.

There was a threshold problem discovered in $\mathrm{Xe}^{131} \mathrm{MAT}=1351, \mathrm{MT}=51$ when $\mathrm{I}$ ran MRKACR. For reasons unknown to me, NJOY is no longer shifting the thresholds for inelastic scattering to insure that

$$
E_{t h} \geq|Q| \frac{A W R+1.0}{A W R} .
$$

Specifically, for MAT=1351, MT=51 $E_{t h}=8.0616400 \mathrm{E}-02$ while $\mathrm{EC}=8.0616423051140 \mathrm{E}-02$. Clearly, $\mathrm{E}$ can be less than EC in the interval $E_{t h} \leq E \leq E C$. I changed EC using EDIT to 8.061600E-02 to eliminate that problem. In subsequent runs with MCNP in which I transported neutrons through $\mathrm{Xe}^{131}$ there were no negative-energy secondary neutrons produced.

There were eleven isotopes $\left(\mathrm{Xe}^{135}, \mathrm{Tc}^{99}, \mathrm{Pr}^{141}, \mathrm{Nd}^{145}, \mathrm{Nd}^{143}, \mathrm{Nd}^{148}, \mathrm{Eu}^{155}, \mathrm{Pm}^{147}, \mathrm{Sm}^{147}, \mathrm{Sm}^{151}\right.$, $\mathrm{Sm}^{152}$ ) for which some of the reaction cross sections were given in terms of the semi-log interpolation INT $=3$ on the ENDF/B-V tapes. This interpolation code $(y$ linear in $\ln x)$ is not handled in MARK because closed-form expressions cannot be written for the integrals. Therefore, I was forced to rely on the ACE/ENDF comparison plots produced with XDATAP to check the NJOY processing of the cross sections for these reactions. The INT $=3$ cross sections have scallops on log-log plots. That only increased my apprehension and I decided to reproduce one of MacFarlane's PENDF tapes starting from "scratch." The deck for that NJOY run is listed here. 


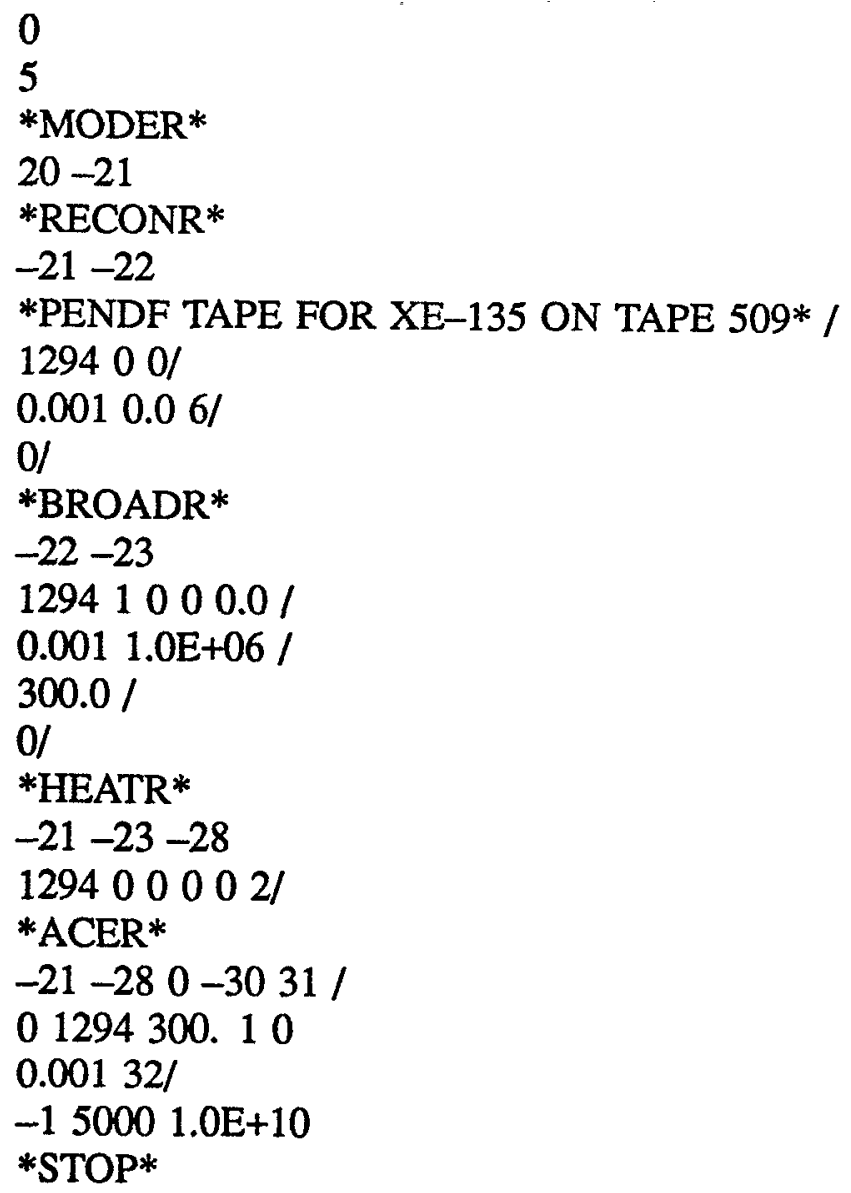

I was processing $\mathrm{Xe}^{135}$ MAT $=1294$ on Tape509. Tape509 was Tape20 in this run. The NJOY outputs for this full run and the "short" run started from the PENDF tape are available in Appendix B. The final ACE files were not identical; I had roughly twice as many points in "my" output ACE file. However, the MRKACR output striped to three and four significant figure agreement and the plots produced in XDATAP run in COMPARE mode were indistinguishable. On the basis of this experience with the one isotope I decided to start my processing from MacFarlane's PENDF tapes - those listed in Table II of Ref. 1. This is not a confession, however, because - after all - I have compared all of the final ACE files on KIDMAN with the original ENDF/B-V cross sections.

Three MCNP runs have been made using KIDMAN. A copy of the first page of each of the runs is included in Appendix C.

I intend to hold on to all the plots and MARK/MRKACR outputs for a while. They are stored in the middle drawer of the filing cabinet in my office under KIDMAN Service Job. The microfiche listings from KIDMAN will be saved to become a permanent addition to our fiche library. 


\section{REFERENCES}

1. R. E. Seamon, "Fission-Product-Isotope Cross Sections in ACE Format for MCNP," Los Alamos National Laboratory internal memorandum to R. B. Kidman (September13, 1989).

2. J. F. Briesmeister, editor, "MCNP - A General Monte Carlo Code for Neutron and Photon Transport Version 3A," Los Alamos National Laboratory manual LA-7396-M, Rev. 2 (September 1986).

3. R. E. MacFarlane, D. W. Muir, and R. M. Boicourt, "The NJOY Nuclear Data Processing System, Volume I: User's Manual," Los Alamos National Laboratory manual LA-9303-M, Vol. I (ENDF-324) (May 1982).

4. R. C. Little, "Cross Section Processing Codes on the CRAY," Los Alamos National Laboratory internal memorandum X-6:RCL $-85-415$ to R. E. Seamon and H. M. Fisher (August 13, 1985).

5. R. C. Little, "The Code CUL," Los Alamos National Laboratory internal memorandum to R.

E. Seamon (February 1, 1984).

6. H. M. Fisher, "XDATAP," Los Alamos National Laboratory report LA-11155-MS (October 1987).

7. R. E. Seamon, "The MARK and MRKACR Codes," Los Alamos National Laboratory internal memorandum X-6:RES-87-182 to Distribution (March 26, 1987).

\section{DISTRIBUTION}

R. C. Little, $X-6$, MS B226

H. M. Fisher, X-6, MS B226

$\mathrm{X}-6$ Files, MS B226 (2)

RES:res 


\section{APPENDIX A}

Listing of CFS File 1076997/MYREDSTUFF/FIXACEFILE

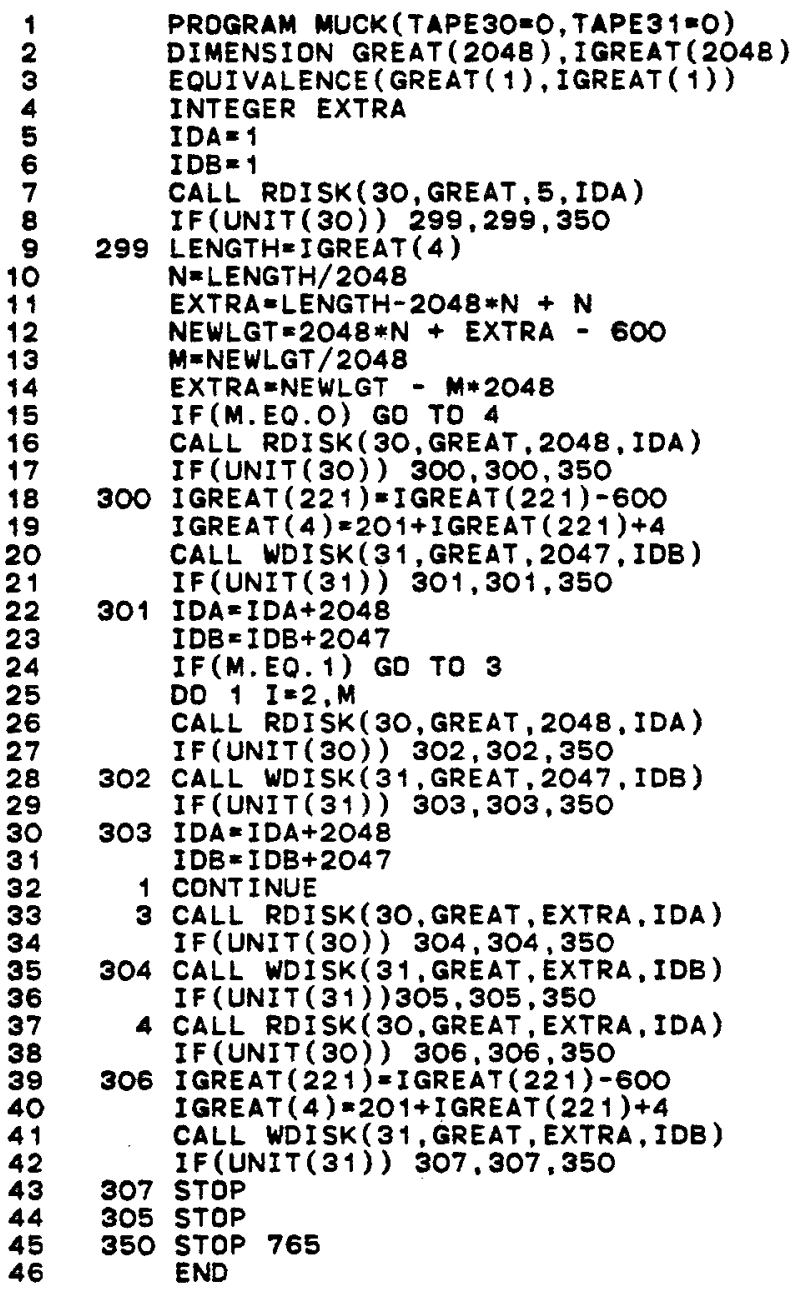




\section{APPENDIX B}

\section{Two NJOY Output Files}

MAT=1294 Straight Through NJOY
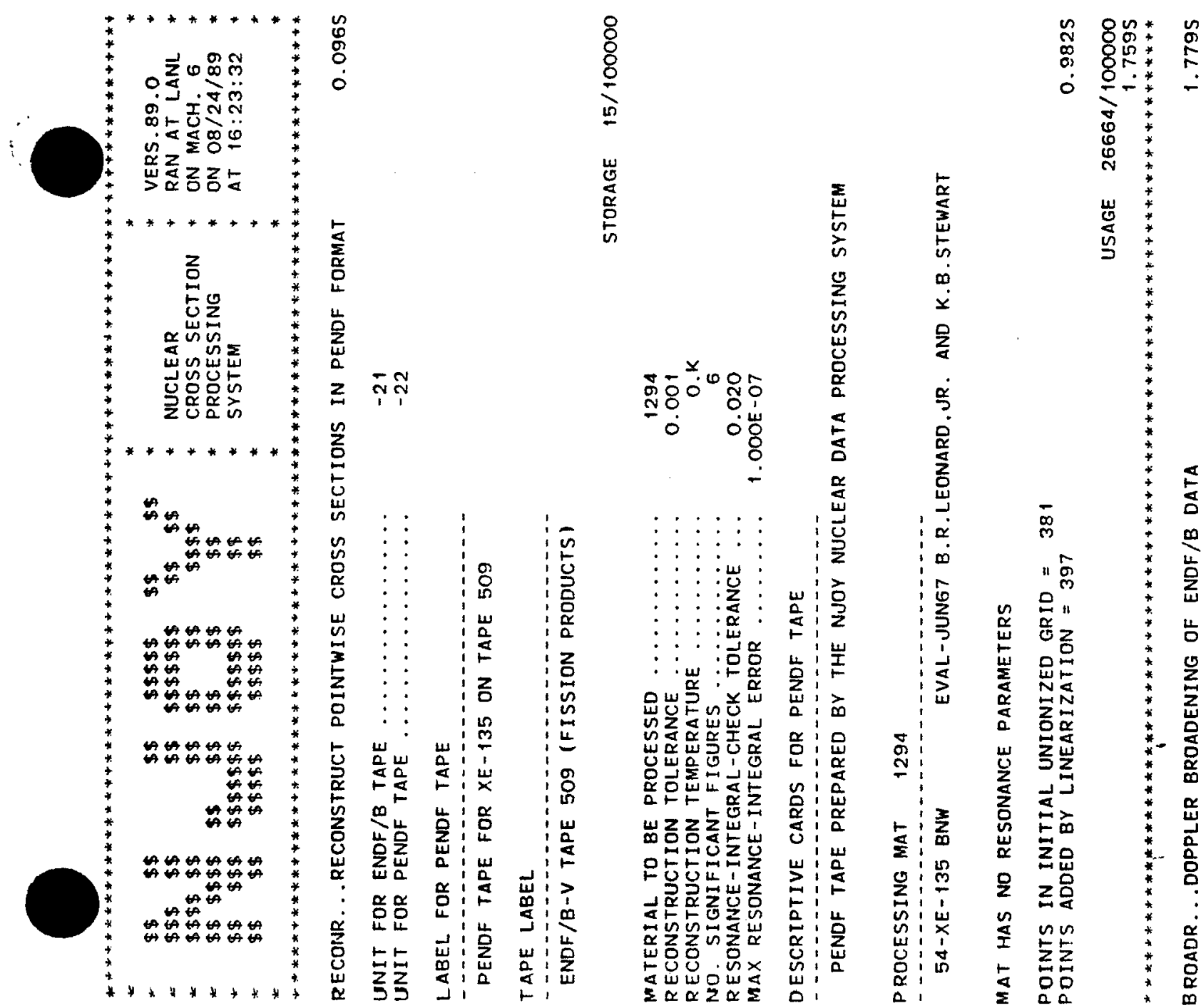

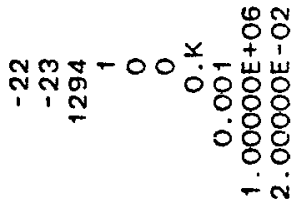

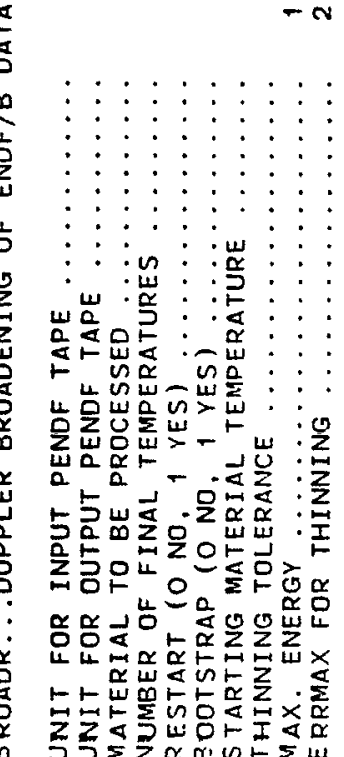




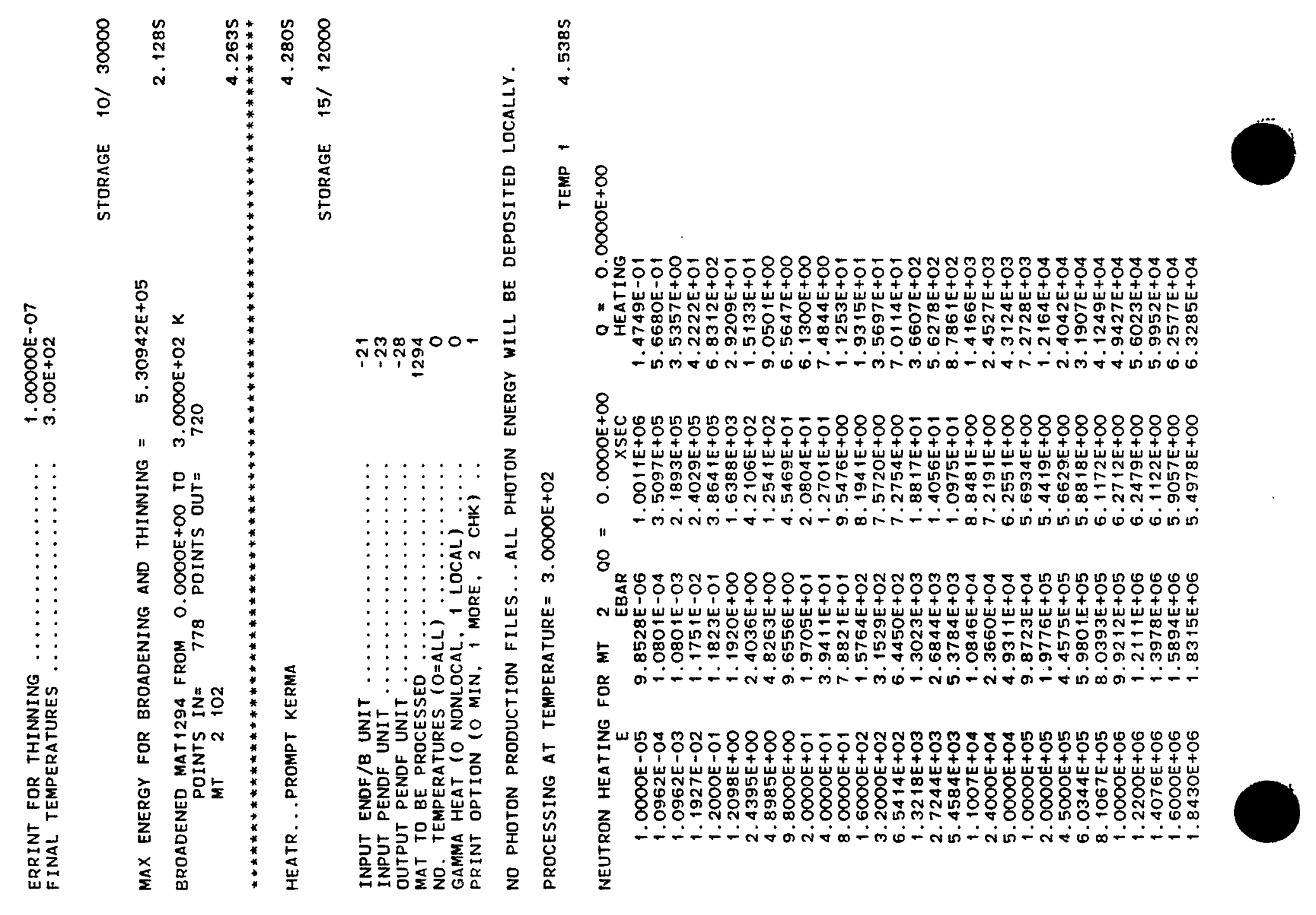




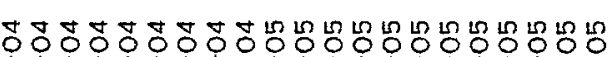

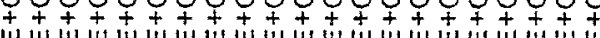

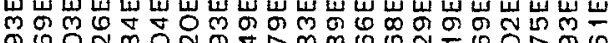

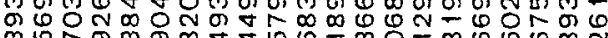
ल

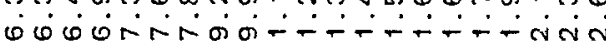

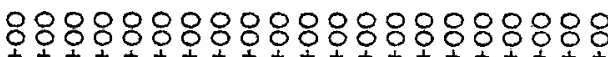

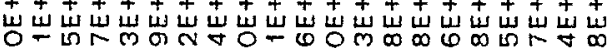
तल का T-

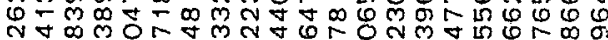

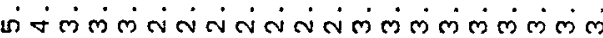

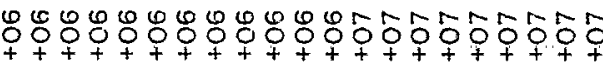

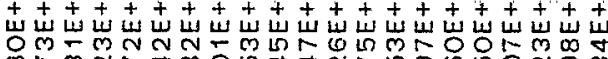

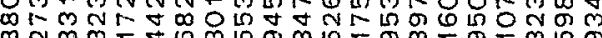

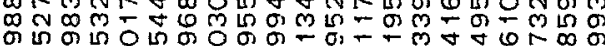
- तरांण

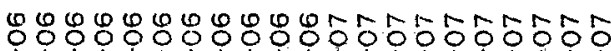

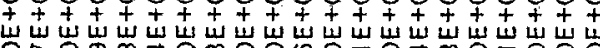

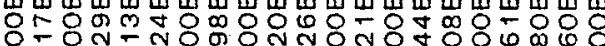

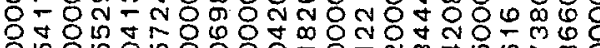
ind

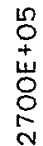

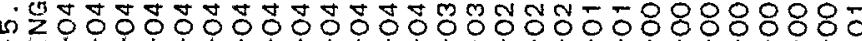

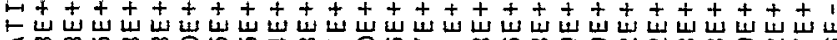
"द m ल 岂士

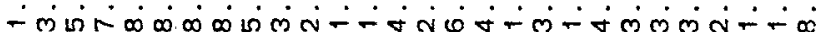

8

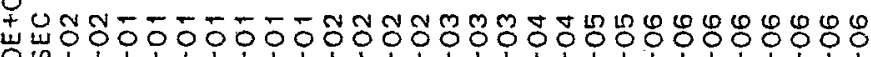
ठㄱ o. रம்--

8

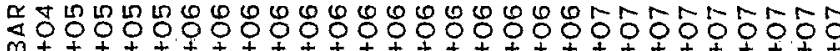

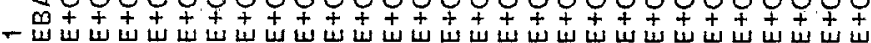
in

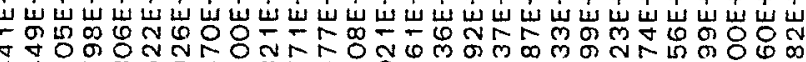
₹

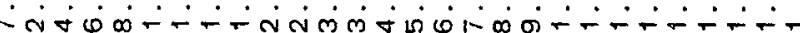

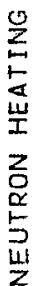

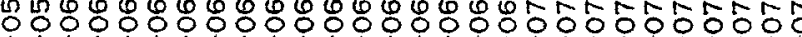

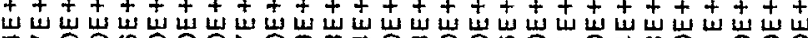

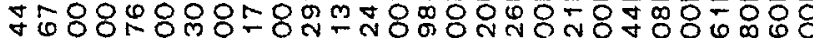

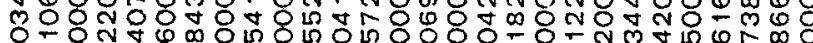
क
10
0
+
0
0
0
0

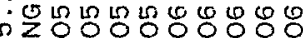

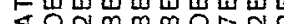

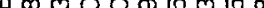
$\cos$

8

सบํํㄷำ$++t$

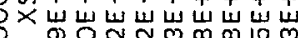
O ণ

is $\dot{\sigma} \dot{0} \dot{\theta} \dot{0} \div \dot{-}-\dot{ }$

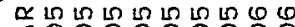

T000000000

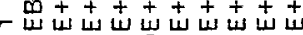
Troor

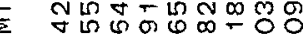
-

2

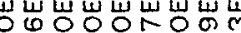
or

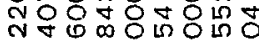
$\therefore \therefore \therefore$ त्लिं 


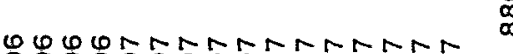

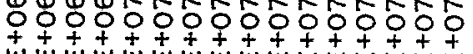

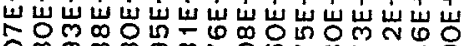

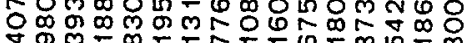

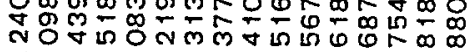

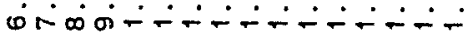

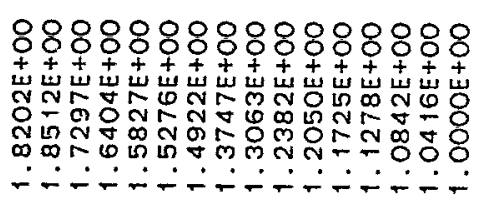

ஜ유유. t t +t+t+t+t+t+t+t+ 出象出山山心

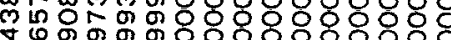

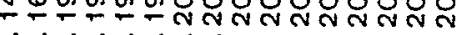

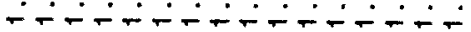

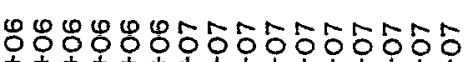

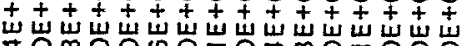

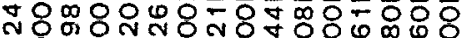

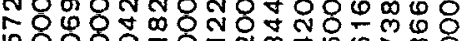
น.
0
0
+
$⿱$
0
0
$\infty$
0

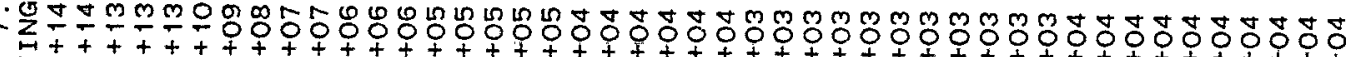

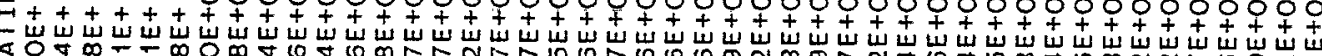

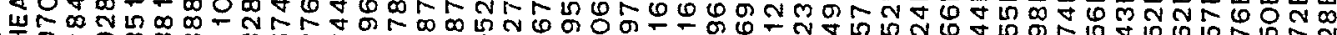

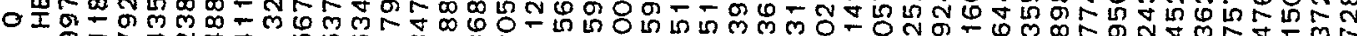
O-r ம $\stackrel{\varphi}{0}$

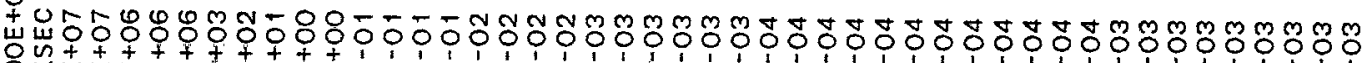

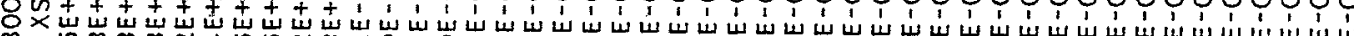

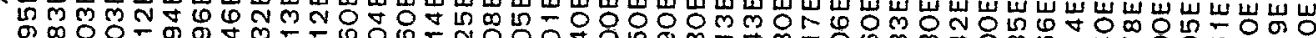
O

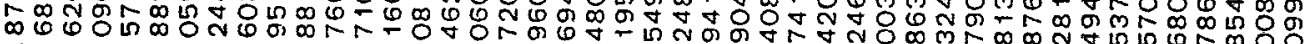
$\infty$ तक

8

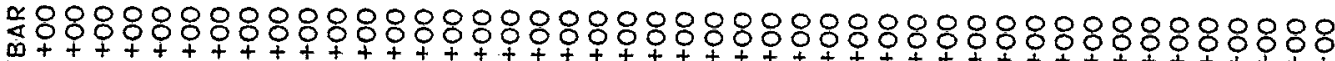

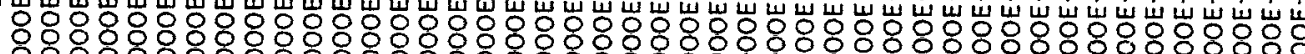

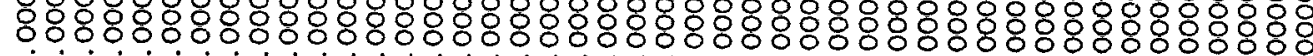

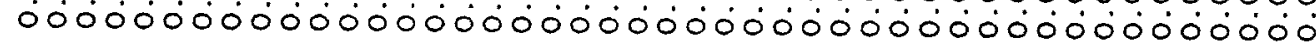
艺w

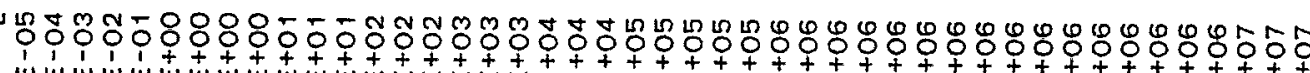
uس

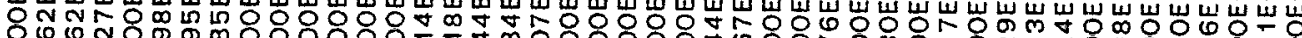
8. - - von 
Oั

山出出山出

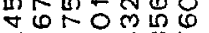

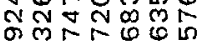

จंเ

웅ㅇㅇㅇㅇㅇㅇㅛ

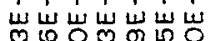

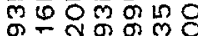

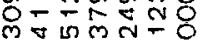
जंत्रतिं
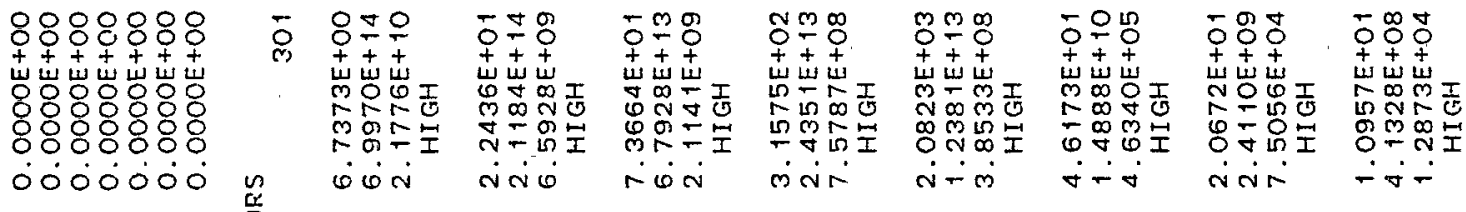

8ㅇํㅇ

웅ํํ 유유

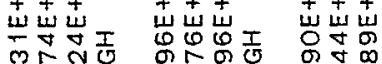

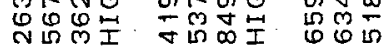

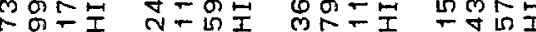

5
5
0
0
0

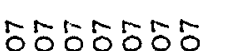

近w

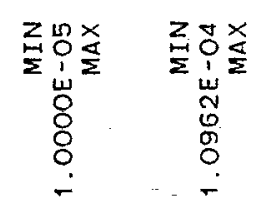

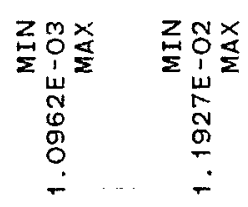

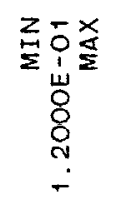

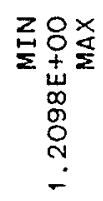
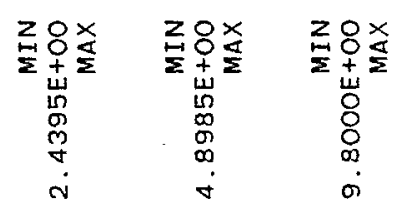

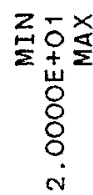

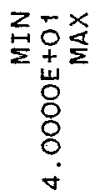




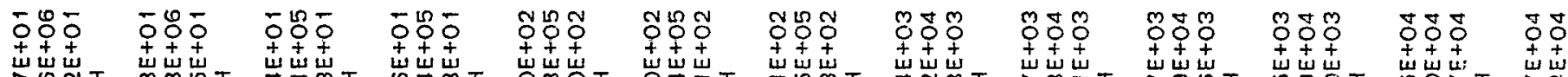

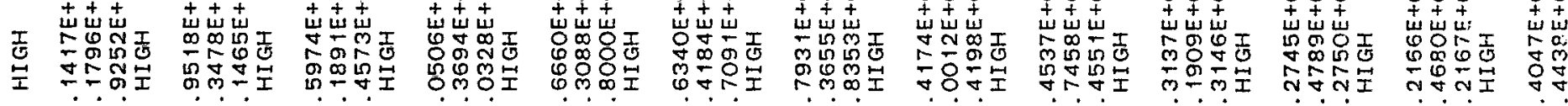
तit $\because \dot{0}$ लंब

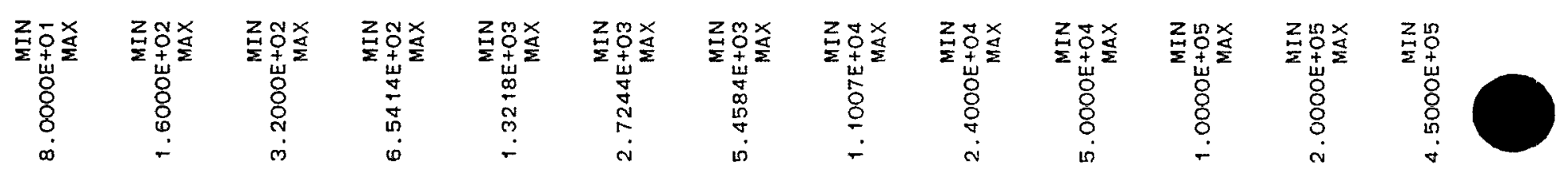





\begin{tabular}{|c|c|c|c|c|c|c|c|c|c|c|c|c|}
\hline 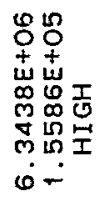 & 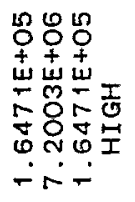 & 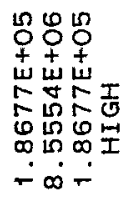 & 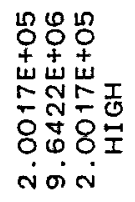 & 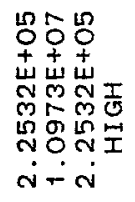 & 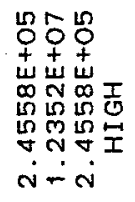 & 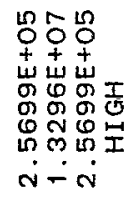 & 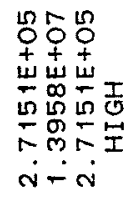 & 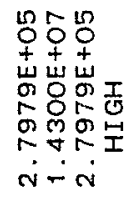 & 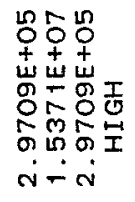 & 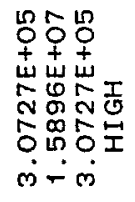 & 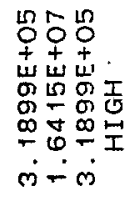 & 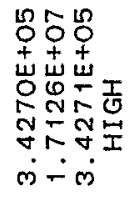 \\
\hline 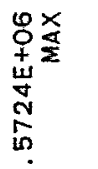 & 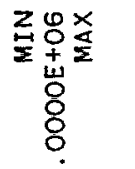 & 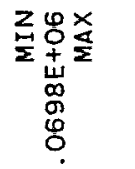 & 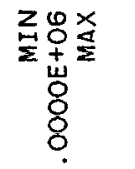 & 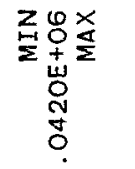 & 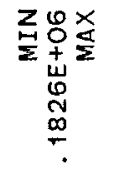 & 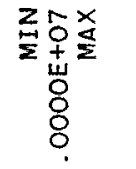 & 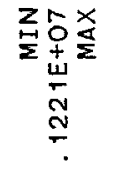 & 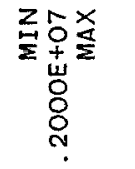 & 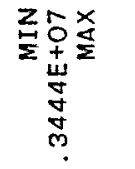 & 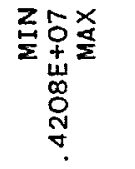 & 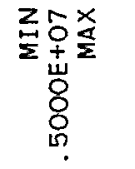 & 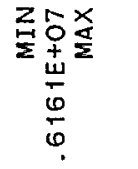 \\
\hline$\dot{0}$ & ن & $\dot{\omega}$ & $\dot{r}$ & $\dot{\infty}$ & $\dot{\sigma}$ & $\therefore$ & - & $\therefore$ & - & $\sigma^{\circ}$ & 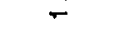 & \\
\hline
\end{tabular}




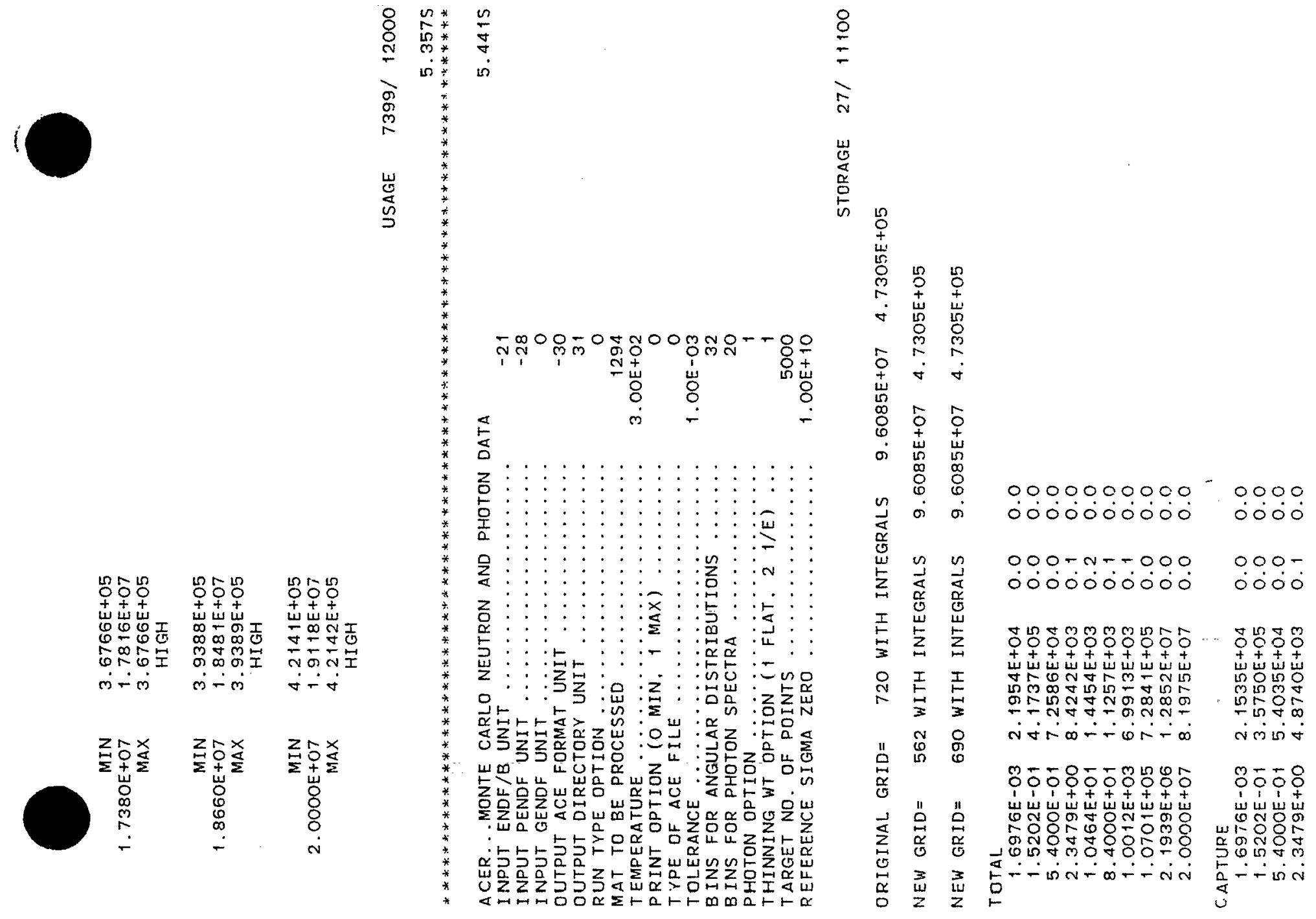


$\stackrel{5}{5}$

$000000 \%$ กับ000 í0ं0்

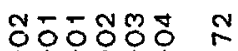

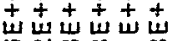

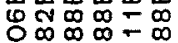

Q

परिण

등ํㅇㅇㅛ

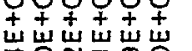
पि口卄 6 잉요잉

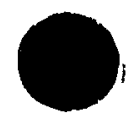



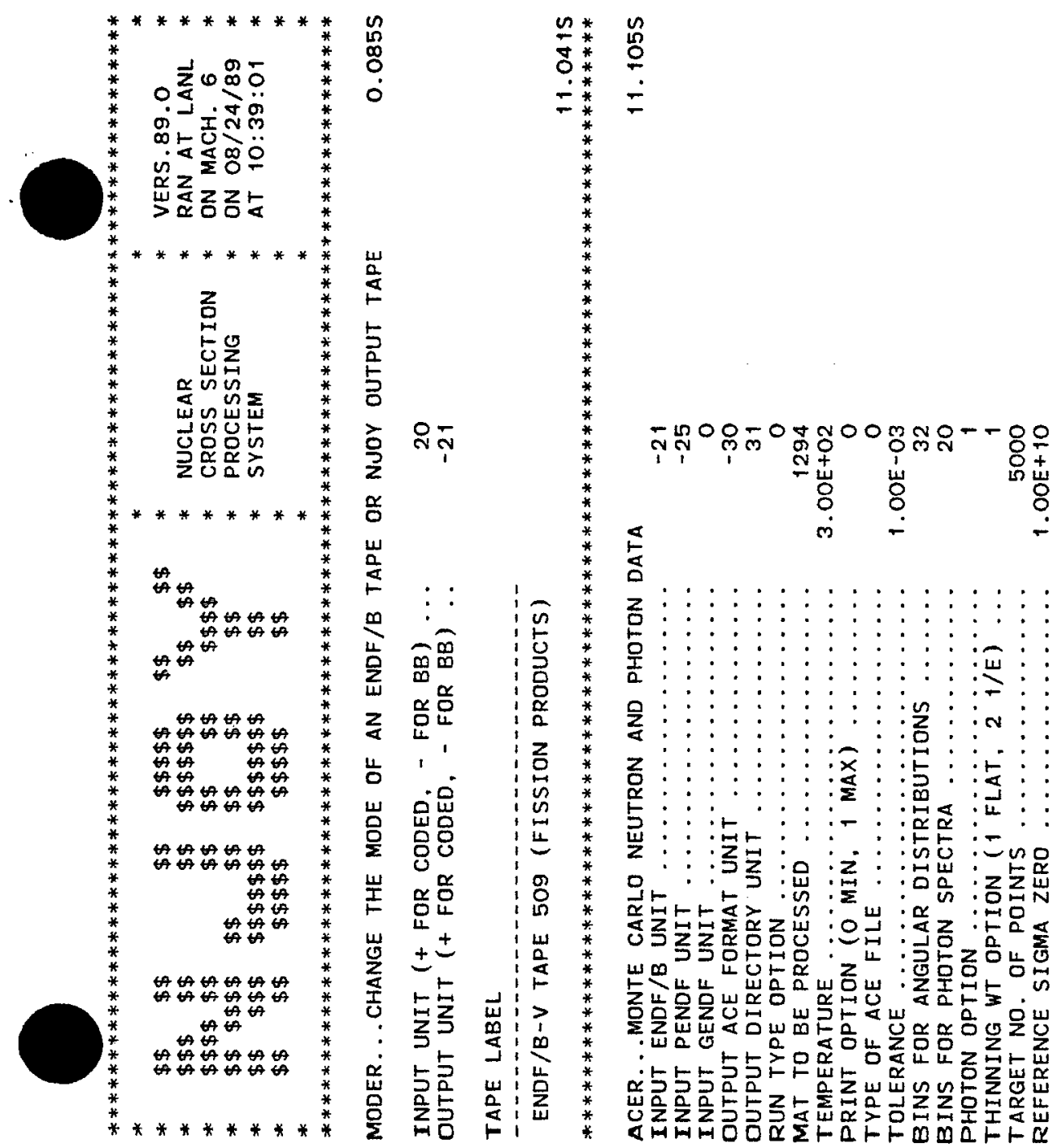

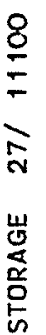

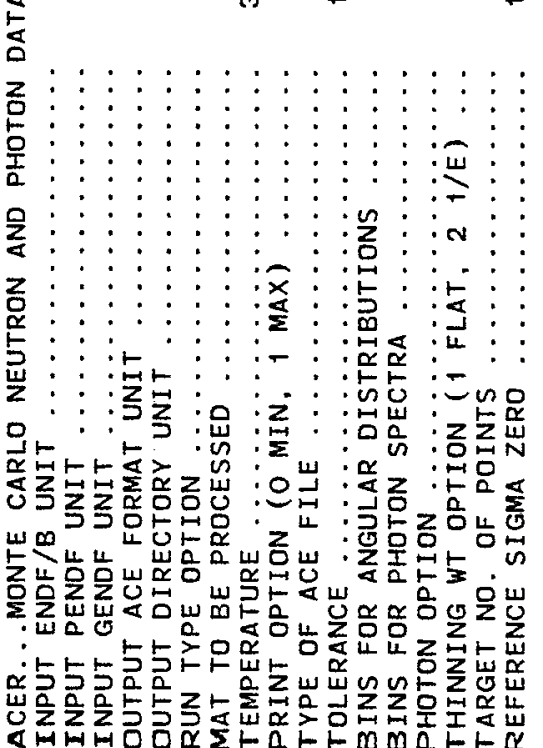

0000000000 00 0000000000 00 $0,00-70000 \quad 00$ 0000000000 0ं

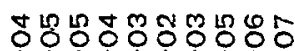

嵌山嵌山嵌山w

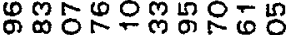

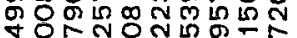

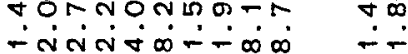
ํํㅇํํㅇํㅇํํํํํำ 
$2.8000 E-01 \quad 2.2563 E+05$

9.4000E-01 1.5266E +04

$4.6000 E+00 \quad 1.9830 E+03$

1.8000E+01 1.683OE+02

1. $6000 \mathrm{E}+02 \quad 5.7074 \mathrm{E}+01$

$2.0000 E+04 \quad 3.5958 E+02$

1.3711E+O6 1.3672E+03

3. $2828 \mathrm{E}+\mathrm{O} 4$

$\begin{array}{ll}0.0 & 0.0 \\ 0.0 & 0.0\end{array}$

0.20 .0

$0.3 \quad 0.0$

$\begin{array}{ll}0.1 & 0.0 \\ 0.1 & 0.0\end{array}$

$0.0 \quad 0.0$

37

39

$39 \quad 39$

$39 \quad 37$

REMOVED $E=1.00000 E+03$ FOR $M F=4 M T=2$ 


\section{APPENDIX C}

\section{Three MCNP Runs for Testing KIDMAN}

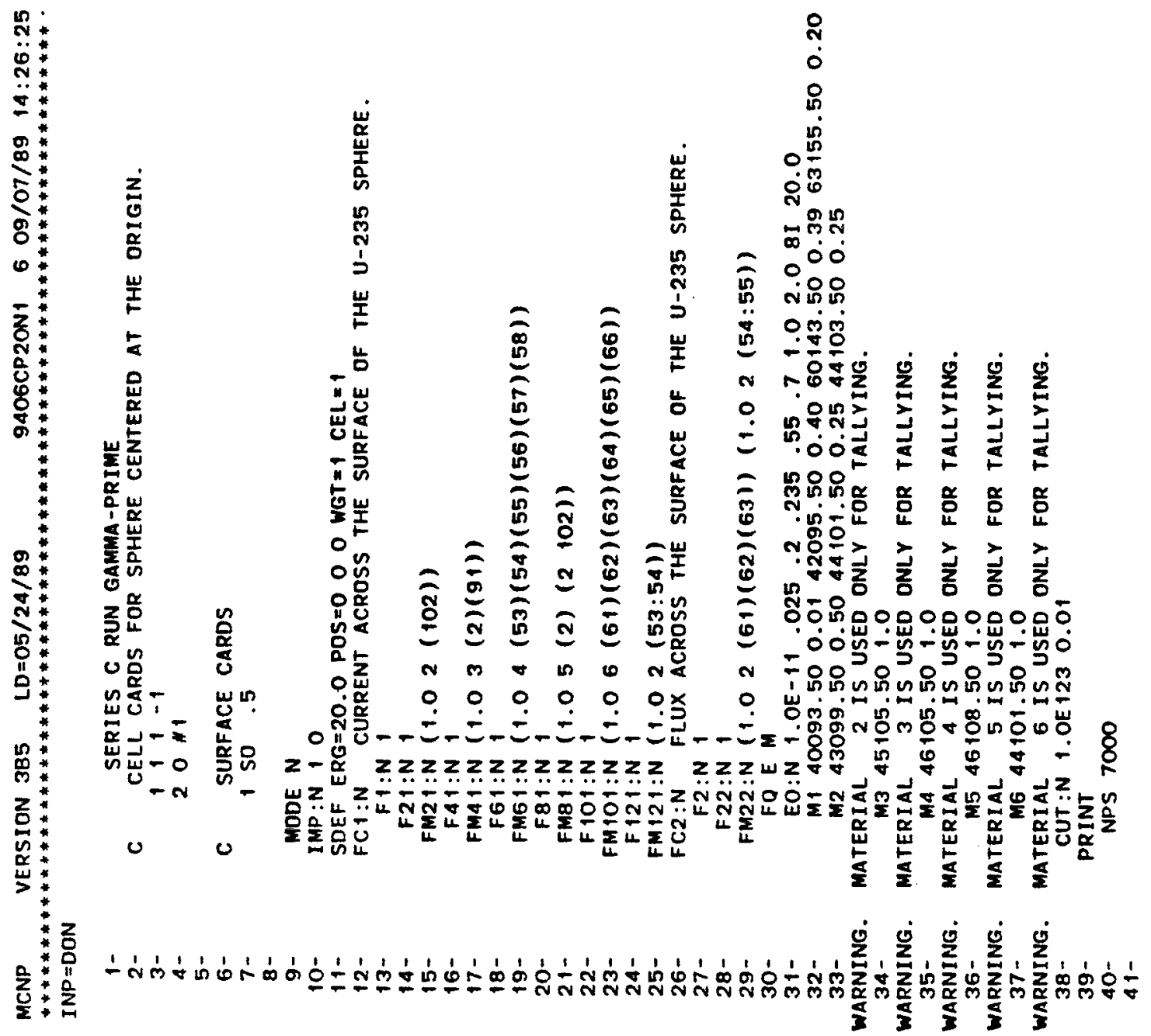




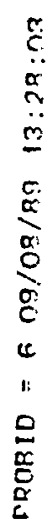

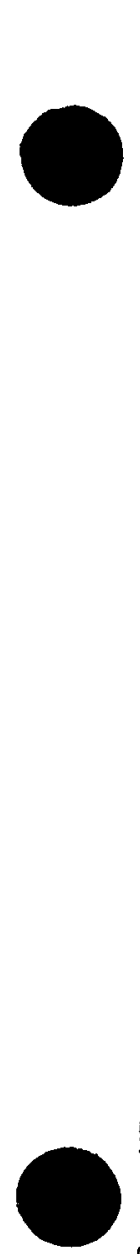

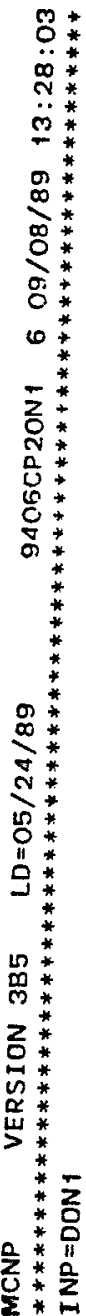

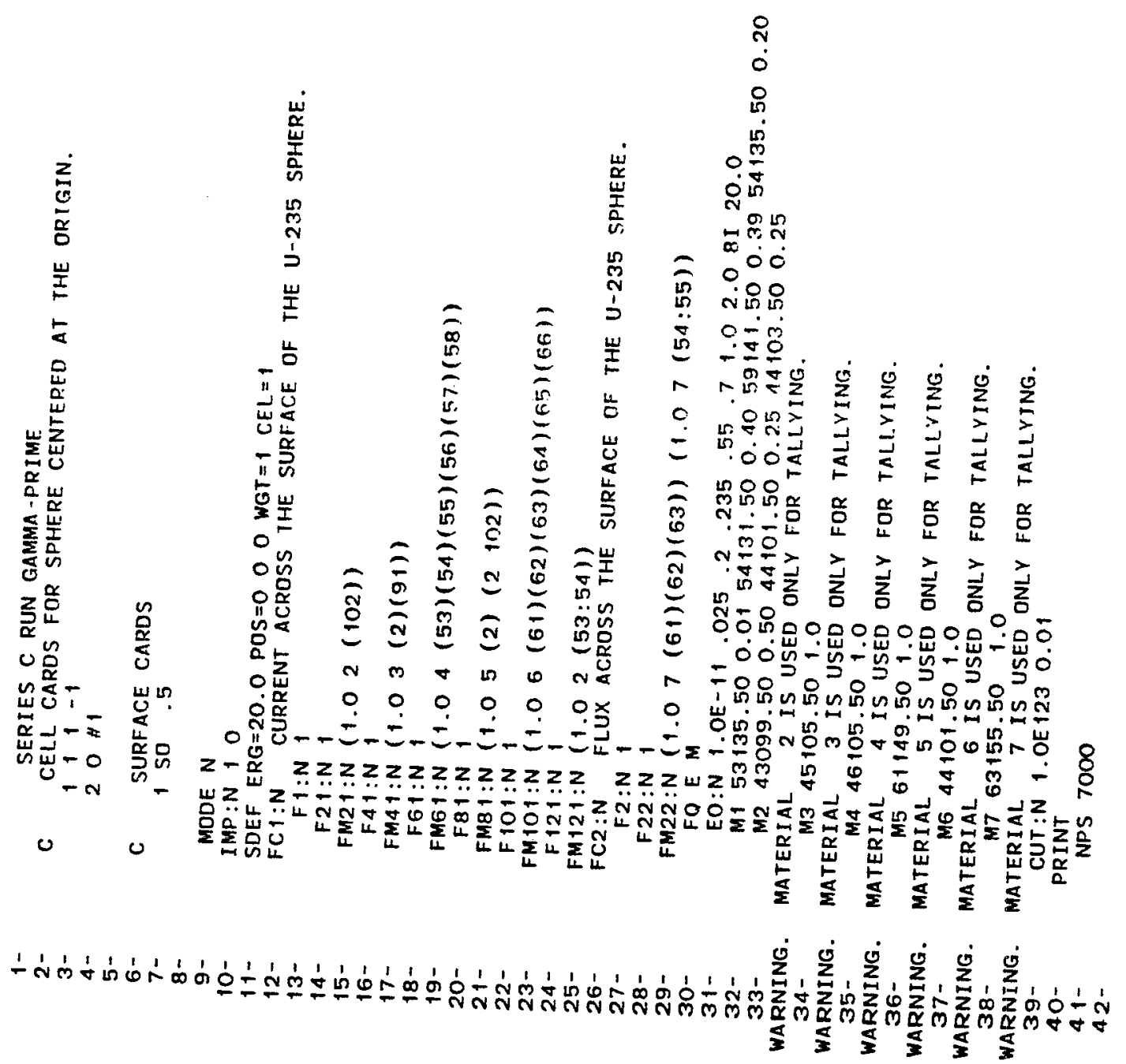


C CELL CARDS FOR SFHERE CENTFPFD AT THE ORIGIN.

$$
\begin{array}{llll}
1 & 1 & 1 & -1 \\
2 & 0 & \# 1
\end{array}
$$

C SURFACE CARDS

$$
1 \text { SO } .5
$$

MODE $N$

MP : $N \perp O$

SDEF ERG $=20.0$ POS $=0$ O O WGT $=1 \quad C E L=1$

FC1:N CURRENT ACROSS THE SUPFACE OF THE U-235 SPHERE.

$F 1: N 1$

$F 21: N 1$

FM21:N (1.02 (102))

F41:N 1

FM41:N (1.03 (2)(91))

FG1:N 1

FM61:N (1.0 4 (53)(54)(55)(56)(57)(58))

F81:N 1

FM81:N (1.05 (2) (2 102))

F101:N 1

FM101:N (1.06(61)(62)(63)(64)(65)(66))

$F 121: N 1$

FM121:N (1.02 $2(53: 54))$

FC2:N FLUX ACROSS THE SURFACE OF THE U-235 SPHERE.

F2:N 1

$F M 22: N(1.02(61)(62)(63))(1.02(54: 55))$

FOE M

EO:N $1.0 E-11$. $025 \quad 2 \quad 2.235 \quad .55 \quad .7 \quad 1.02 .08 \mathrm{I} 20.0$

$\begin{array}{lllllllllllll}31- & E O: N 1.0 E-11 & .025 & .2 & 235 & .55 & 7 & 1.0 & 2.0 & 81 & 20.0 & & \\ 32- & M 1 & 62147.50 & 0.20 & 62151.50 & 0.20 & 62150.50 & .2 .62152 .5 & .2 & 63155.5 & .2\end{array}$

33- M2 60143.5 .25 60145.5 25 511485.25611495 .25

WARNING. MATERIAL 2 IS USED ONLY FOR TALLYING.

34- M3 60147.50 1.0

WARNING. MATERIAL 3 IS USED ONLY FOR TALLYING.

35- M4 60148.501 .0

WARNING. MATERIAL 4 IS USED ONLY FOR TALLYING.

36- M5 61149.50 1.0

WARNING. MATERIAL 5 IS USED ONLY FOR TALLYING

37- M6 59141.501 .0

WARNING. MATERIAL 6 IS USED ONLY FOR TALLYING.

$38-$

$39-$

40-

CUT:N 1.OE123 0.01

PRINT

NPS 7000 